\title{
Initial clinical experience of ranibizumab therapy for neovascular age-related macular degeneration
}

This article was published in the following Dove Press journal:

Clinical Ophthalmology

9 November 2010

Number of times this article has been viewed

\section{Tryfon Rotsos \\ Praveen J Patel \\ Fred $\mathrm{K}$ Chen \\ Adnan Tufail}

Medical Retina Service, Moorfields Eye Hospital, London, UK
Correspondence: Tryfon Rotsos Medical Retina Service, Moorfields Eye Hospital, I62 City Road, London, ECIV 2PD, UK

Tel +306974727279

Fax +3021075I 7764

Email tryfonrotsos@hotmail.com
Purpose: To describe the visual acuity and safety outcomes for the first 50 patients with neovascular age-related macular degeneration (nAMD) treated with ranibizumab at Moorfields Eye Hospital.

Methods: A retrospective analysis of case notes from the first 50 consecutive patients with Primary Care Trust funding for ranibizumab therapy for nAMD. Visual acuity outcomes and adverse events were noted, as were service delivery-related indicators.

Results: The mean ( \pm standard deviation) age of the 50 patients was $81 \pm 17$ years. The mean follow-up of patients was $13.6 \pm 2$ (range 7.7-18) months. The mean change in visual acuity \pm standard error was $+4.6 \pm 2.2$ letters at the end of follow-up, with $26 \%$ gaining 15 letters or more. The mean (median) number of injections was 4.7 (4.5) per 12-month period. The mean (median) delay in Primary Care Trust funding approval was 35 days (32 days) prior to the final appraisal document from the National Institute of Health and Clinical Excellence. Conclusions: The real-world outcomes of ranibizumab therapy in this initial cohort of patients with nAMD are comparable with those reported in the pivotal, randomized, controlled trials using fewer injections and a prn strategy of retreatment to achieve the gain in visual acuity.

Keywords: macular degeneration, retina, ranibizumab

\section{Introduction}

Ranibizumab (Lucentis ${ }^{\circledR}$; Novartis, Basel, Switzerland) therapy has transformed the treatment of choroidal neovascularization due to age-related macular degeneration, ie, neovascular AMD (nAMD). Licensed for use by the European Medicines Agency in 2006, ranibizumab is an affinity-matured monoclonal antibody fragment which binds to and blocks the action of all isoforms of vascular endothelial growth factor and is administered by four-weekly intravitreal injection. The pivotal Phase III studies used a continuous dosing strategy. ${ }^{1,2}$ However, the resource and patient burden of monthly injections led clinicians to investigate other treatment strategies. The PrONTO (Prospective Optical Coherence Tomography Imaging of Patients with Neovascular AMD Treated with intraOcular Ranibizumab) study was a prospective, open-label study in which patients with subfoveal choroidal neovascularization and a minimum optical coherence tomography (OCT) retinal thickness of $300 \mu \mathrm{m}$ were given three injections of ranibizumab on a monthly basis, with further retreatment based on physician-driven, OCT-guided retreatment criteria using monthly follow-up. ${ }^{3,4}$ Patients were still followed up every four weeks in this study. This treatment strategy was also used by the National Institute of Health and Clinical Excellence (NICE) when determining the cost-effectiveness of ranibizumab therapy, with an average 
of six injections in the first year of therapy. Although the PrONTO study followed up patients on a four-weekly cycle, an alternative approach is the "inject and extend" strategy ${ }^{5}$ in which the interval between patient visits is increased in the absence of disease activity, with retreatment based on OCT criteria. However, there are very few reports of outcomes of ranibizumab therapy using prn strategies in clinical practice, and it remains unclear how the outcomes of clinical trials relate to outcomes in clinical practice.

Because continuous four-weekly review of patients as per clinical trial paradigms may not be practical or achievable for all elderly patients with nAMD, it is important to report real-life outcomes of ranibizumab therapy for patients in the clinic. The provision of ranibizumab funding from Primary Care Trusts (PCTs) in England and Wales was on a caseby-case basis prior to definitive NICE guidance. This led to delays in treatment resulting from funding delays and service provision-related factors. This retrospective case series also provided an opportunity to report key outcome measures in service delivery which may be used to generate benchmark indicators of service delivery and quality for the delivery of nAMD treatment services.

There is therefore a need to report outcomes of ranibizumab therapy for nAMD in clinical practice. The aim of this study was to report outcomes of ranibizumab therapy in the first cohort of patients funded for the drug at a large London teaching hospital, and reporting both visual acuity outcomes and service provision-related outcomes.

\section{Materials and methods}

This was a retrospective case note review of the first 50 patients with PCT funding for ranibizumab therapy at Moorfields Eye Hospital. Applications to the PCTs for ranibizumab funding were made if the lesion characteristics and visual acuity of the eye being considered for treatment fulfilled the inclusion and exclusion criteria for the ANCHOR (ANti-VEGF Antibody for the Treatment of Predominantly Classic CHORoidal Neovascularization in AMD) and MARINA (Minimally classic/occult trial of the Anti-VEGF antibody Ranibizumab In the treatment of Neovascular AMD) Phase III trials at the time of initial funding application. However, patients differed from the pivotal ranibizumab trials in that many had received previous treatment for nAMD (including antivascular endothelial growth factor therapy). The decision to apply for funding was determined on a case-by-case basis at weekly consultant meetings. Any delays in funding and treatment may lead to a change in lesion composition, with the potential for subretinal fibrosis or retinal pigment epithelium atrophy to compromise outcomes. Patients were identified from the funding database and case notes were obtained to confirm the diagnosis. If both eyes had obtained funding for treatment, the first eye undergoing treatment was included. Patients included in this study predated the definitive NICE appraisal document issued in August 2008. We included patients with previous treatment in this cohort as a representative case mix of our initial experience.

The date of the initial funding application, notification of successful funding, and dates of patient visits were all noted. The angiographic classification of the choroidal neovascularization was also noted from the patient notes, as was whether a ranibizumab injection was given at each visit. Visual acuity at each visit was noted as an ETRS (Early Treatment of Diabetic Retinopathy) score, as were any ocular or systemic adverse events. OCT was performed at each visit and prn treatment based on OCT-guided retreatment criteria (PrONTO study) was carried out. The development of subretinal fibrosis, retinal pigment epithelium atrophy, macular hemorrhage, or a retinal pigment epithelium tear was noted by the treating ophthalmologist because these may compromise visual acuity outcomes.

\section{Statistical analysis}

Visual acuity results are reported as mean and median values using the Student's $t$-test and Wilcoxon signed-rank test to compare values. The influence of previous treatment on mean and median visual acuity change was also examined. Correlations between variables were assessed using both the Pearson correlation and Spearman nonparametric correlation analyses.

The influence of baseline visual acuity on the number of injections over 12 months (calculated from the number of reinjections received and the follow-up duration) was assessed, as was the influence of the number of injections per 12 months on visual acuity outcomes. In addition, the correlation between visual acuity change and duration of follow-up was also examined with a scatter plot and both the Pearson correlation and Spearman nonparametric correlation analyses.

\section{Results}

Data from 50 eyes of 50 patients were available for analysis. There were $30(60 \%)$ females and $20(40 \%)$ males. The mean ( \pm standard deviation) age of patients was $80 \pm 17$ years, with $26(54 \%)$ being right eyes and 24 (48\%) left eyes. There were five eyes with classic no occult or predominantly classic 
lesions and 45 eyes with minimally classic lesions or occult no classic lesions. Twenty-eight eyes (56\%) had received previous treatment for nAMD (12 eyes [24\%] had received bevacizumab intravitreal injection and 16 eyes [32\%] had received photodynamic therapy). The mean and median interval for ranibizumab funding approval from PCTs was 35 days and 32 days, respectively. At baseline (prior to ranibizumab therapy), the mean and median visual acuity ETDRS letter scores were $46(6 / 38)$ and 50 (6/30), respectively. The initial treatment paradigm used was three injections then prn in 28 eyes (56\%), two injections then prn in 18 eyes $(36 \%)$, and one injection then prn in four eyes $(8 \%)$. There were outcome differences in the three groups, with a trend for better outcomes with three injections + prn (mean +8.3 letter gain in this group versus +0.8 letters in $2+$ prn and -3.8 letters in $1+$ prn). However, it is difficult to draw definitive conclusions about the merits of the three injections + prn dosing schedule because most patients on the other two dosing schedules had received previous treatment and were more likely to have more chronic lesions. Furthermore, the subgroups were small in number.

The mean and median number of visits was $11 \pm 2.3$ and 10 (range 6-15), respectively, with a mean and median follow-up duration of $13.6 \pm 2.5$ (range 7.7-18) months. For the 37 eyes of 37 patients (74\%) with 12 months or more of follow-up, the mean number of visits in the initial 12-month treatment period was 9.8 . There were 28 patients who had received previous treatment for nAMD in the index eye, and this modified the need for the initiation of ranibizumab therapy, with some patients not needing immediate treatment due to stabilization of nAMD, leading to an increase in the interval between funding approval and treatment. For the 22 patients with no previous treatment, the mean and median delay in the first ranibizumab injection after funding was obtained was 21 days and 20 days, respectively. There were nine patients of the $22(41 \%)$ who received treatment in two weeks or less, with four patients waiting longer than one month after PCT funding approval notification.

During the course of treatment, there was an improvement in the mean and median visual acuity scores of +4.6 letters (Student's $t$-test, $\mathrm{t}=2.07, P=0.04$ ) and +5 letters (Wilcoxon signed-rank test, $\mathrm{z}=-1.96 ; P=0.05$ ), respectively. Figure 1 shows the distribution of visual acuity change for this cohort of patients. Table 1 summarizes the visual acuity outcomes both at six months and at final follow-up.

Of note, 42 patients (84\%) lost less than 15 letters (three lines) of visual acuity (95\% confidence interval [CI]: $79 \%-89 \%$ ) and 13 patients (26\%) gained 15 letters or more in visual acuity (95\% CI: 14\%-38\%) at the end of follow-up. The reasons for vision loss of 15 letters or more in the eight patients were development of subfoveal/subretinal fibrosis in four patients, and geographic atrophy in two patients. The reason for vision loss in the remaining two patients was not listed.

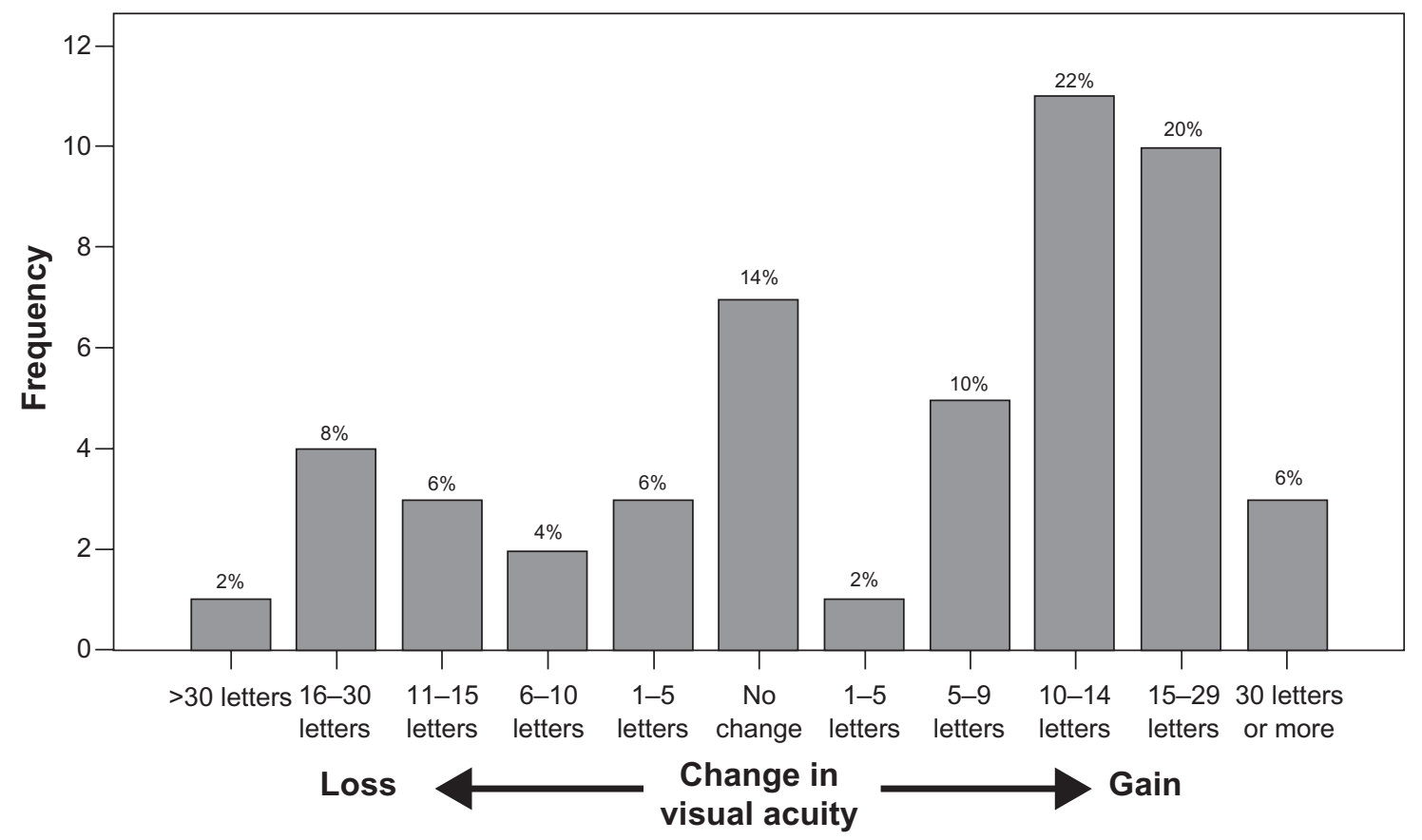

Figure I Histogram showing change in visual acuity after ranibizumab therapy. 
Table I Summary of visual acuity outcomes

\begin{tabular}{lll}
\hline Visual acuity outcomes & At 6 months & At final follow-up \\
\hline Mean change (SE) & $+6.4( \pm I .4)$ & $+4.6( \pm 0.7)$ \\
Median change & +5 & +5 \\
Number of patients (\%) & & \\
$\quad$ Losing less than I5 letters & $48(96 \%)$ & $42(84 \%)$ \\
Losing less than I0 letters & $45(90 \%)$ & $40(80 \%)$ \\
Avoiding any loss of visual & $44(88 \%)$ & $37(74 \%)$ \\
acuity ( $\geq 0$ letter change) & & \\
Gaining I0 letters or more & $21(42 \%)$ & $24(48 \%)$ \\
Gaining I5 letters or more & $14(28 \%)$ & $13(26 \%)$ \\
\hline
\end{tabular}

The visual acuity outcomes were obtained with a mean and median number of treatments of $5.3 \pm 2.5$ and 5.0, respectively, over the total follow-up period. When the number of treatments was standardized for the duration of follow-up, this equated to a mean and median number of treatments per year of $4.7 \pm 1.9$ and 4.5 , respectively. For the 37 patients with at least one year of follow-up, the mean and median numbers of treatments per year were $4.7 \pm 2.1$ and 4.5 , respectively.

No correlation was found between number of injections over 12 months and baseline visual acuity (Pearson, $r=0.06$; $P=0.70 ;$ Spearman, $\mathrm{r}=0.05 ; P=0.74)$. We did not carry out an analysis of the correlation between baseline lesion angiographic subtype and number of treatments because there were too few classic no occult or predominantly classic lesions to permit a meaningful analysis. No correlation was found between number of injections over 12 months and change in visual acuity at final follow-up (Pearson, $\mathrm{r}=0.18$; $P=0.21$; Spearman, $\mathrm{r}=0.06 ; P=0.70$ ). In addition, as Figure 2 demonstrates, no correlation was found between change in visual acuity and length of follow-up (Pearson, $\mathrm{r}=0.17 ; P=0.25$; Spearman, $\mathrm{r}=0.09 ; P=0.54$ ).

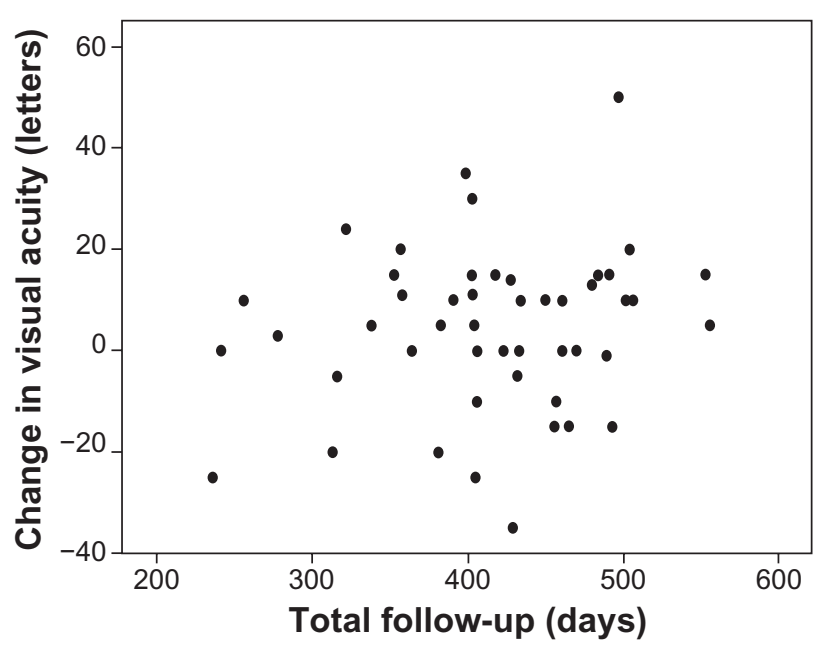

Figure 2 Plot of change in visual acuity against length of follow-up.
The mean change in visual acuity in 28 patients who had received previous treatment was +2.8 letters compared with a mean gain of +6.9 letters in 22 patients with no previous treatment (Student's $t$-test, $P=0.36$ ). The median change in visual acuity in patients who had received previous treatment was +7.5 letters compared with a gain of +5.0 letters in patients with no previous treatment (Wilcoxon rank-sum test, $P=0.82$ ).

\section{Adverse events}

Ranibizumab therapy was well tolerated in this cohort of patients, with no reports of endophthalmitis, traumatic cataract, or retinal detachment. In addition, there were no thromboembolic events reported in this cohort of patients during follow-up.

\section{Discussion}

This report describes the outcome of ranibizumab therapy in the first cohort of patients funded for treatment at a tertiary care centre in the UK. These patients were treated at a time before the publication of NICE guidance regarding ranibizumab and pegaptanib therapy, when funding of therapy by Primary Care Trusts was problematic and often at the discretion of an exceptional case panel. This led to delays in treatment which were compounded by the lack of capacity for intravitreal treatment. Despite these barriers to treatment and optimal outcomes, the results are comparable with the outcomes seen in the pivotal Phase III trials of ranibizumab. ${ }^{1,2}$ The treatment strategy adopted in the randomized controlled trials was one of continuous therapy but, in view of the patient- and resource-intensive nature of monthly intravitreal injection, investigators have used a modified treatment strategy with monthly visits but with three loading doses and further prn treatment based on OCT-guided retreatment criteria (PrONTO study). ${ }^{3}$ Two-year data are now available for this treatment strategy, suggesting comparable results with continuous dosing but with a mean number of treatments of 5-6 per year of follow-up, with monthly visits. ${ }^{4}$ Others have described an inject and extend model in which the follow-up interval after the three-monthly loading injections was modified and extended if disease quiescence was achieved. ${ }^{5}$ The advantage of this approach is to reduce the number of visits to the hospital eye clinic, an important consideration in this group of elderly patients with other comorbidity. Given the resource limitations and the disease burden, although ophthalmologists in the UK at the beginning of ranibizumab therapy started with a fixed monthly review interval as per the PrONTO study, the 
pressures of service delivery and patient comorbidity led to an increasing number of patients funded for treatment potentially receiving less frequent follow-up, shifting to a more treat and extend approach. Our cohort reflects this shift only to a limited degree because the mean and median patient visits in 12 months were approximately 10, suggesting reasonably close follow-up of patients, without much extension in follow-up interval. This may go some way to explaining the good outcomes we report.

In this study, we found no relationship between baseline visual acuity or the number of injections and visual acuity gain. This may reflect the fact that patients with poor baseline visual acuity may have had previous treatment with subretinal fibrosis or atrophy. The scatter plot (Figure 2) and the analyses performed show no correlation between change in visual acuity and length of follow-up in this cohort, suggesting no fall-off in visual acuity gains with increased follow-up.

Our results contrast with those from another recent retrospective study by Cohen et al from a tertiary care center in France. ${ }^{6}$ We report a mean gain in visual acuity (+4.6 letters) while the previous study reported only stabilization in visual acuity (mean gain of 0.7 letters). Both the previous study and our report describe real-world use of ranibizumab in different health care settings. Although there were potentially more delays in treatment for the cohort of patients we describe, in view of the delay in authorizing funding for ranibizumab therapy from PCTs, our outcomes appear better than those reported by Cohen et al. This may be in view of the larger number of treatments and the closer follow-up of patients in our series.

Because there are difficulties in directly translating the results from clinical trials into clinical practice, it is reassuring to report outcomes in this cohort of patients not dissimilar to those in the Phase III ranibizumab studies. Further work is needed to determine whether more prompt treatment may improve outcomes. In this study we describe benchmark indicators of service delivery, including the time from PCT funding to treatment. The Royal College of Ophthalmologists recommends a diagnosis to treatment time of up to two weeks, and indeed 14 days was the duration of the screening period for patients included in the randomized controlled ranibizumab trials. We report positive outcomes, despite only $41 \%$ of patients receiving treatment within 14 days of funding, with a mean delay of 35 days in obtaining funding for therapy from the PCTs.

The limitations of this study include its retrospective nature and the nonstandardized follow-up or retreatment strategy, although this may also be viewed as a potential advantage, reflecting real-world use of this agent prior to NICE guidance. The advantages include the report of realworld outcome data for the use of ranibizumab in the treatment of nAMD with additional data regarding the time taken to start therapy and the use of standardized visual acuity measurement in assessing outcomes. Further studies are needed to determine what factors are most important in achieving good outcomes in clinical practice. In summary, this is the first study to report positive outcomes for the use of ranibizumab in the treatment of nAMD in a clinical setting.

\section{Disclosure}

AT has been on the advisory boards for the following companies involved in research or products related to the treatment of retinal disorders: Novartis, Pfizer, GSK, MSD, and Allergan. PP has received travel grants from Novartis. The other authors report no competing interests in this work.

\section{References}

1. Brown DM, Kaiser PK, Michels M, et al. Ranibizumab versus verteporfin for neovascular age-related macular degeneration. $N$ Engl J Med. 2006;355:1432-1444.

2. Rosenfeld PJ, Brown DM, Heier JS, et al. Ranibizumab for neovascular age-related macular degeneration. $N$ Engl J Med. 2006;355:1419-1431.

3. Fung AE, Lalwani GA, Rosenfeld PJ, et al. An optical coherence tomography-guided, variable dosing regimen with intravitreal ranibizumab (Lucentis) for neovascular age-related macular degeneration. Am J Ophthalmol. 2007;143:566-583.

4. Lalwani GA, Rosenfeld PJ, Fung AE, et al. A variable-dosing regimen with intravitreal ranibizumab for neovascular age-related macular degeneration: Year 2 of the PrONTO Study. Am J Ophthalmol. 2009;148: 43-58.

5. Spaide R. Ranibizumab according to need: A treatment for age-related macular degeneration. Am J Ophthalmol. 2007;143:679-680.

6. Cohen SY, Dubois L, Tadayoni R, et al. Results of one-year's treatment with ranibizumab for exudative age-related macular degeneration in a clinical setting. Am J Ophthalmol. 2009;148:409-413.
Clinical Ophthalmology

\section{Publish your work in this journal}

Clinical Ophthalmology is an international, peer-reviewed journal covering all subspecialties within ophthalmology. Key topics include: Optometry; Visual science; Pharmacology and drug therapy in eye diseases; Basic Sciences; Primary and Secondary eye care; Patient Safety and Quality of Care Improvements. This journal is indexed on

\section{Dovepress}

PubMed Central and CAS, and is the official journal of The Society of Clinical Ophthalmology (SCO). The manuscript management system is completely online and includes a very quick and fair peer-review system, which is all easy to use. Visit http://www.dovepress.com/ testimonials.php to read real quotes from published authors. 